\title{
Modeling the Transmission Dynamics of Bovine Tuberculosis
}

\author{
Theresia Shirima Sabini $\mathbb{D}^{1},{ }^{1}$ Jacob Ismail Irunde, ${ }^{2}$ and Dmitry Kuznetsov ${ }^{1}$ \\ ${ }^{1}$ School of CoCSE, The Nelson Mandela African Institution of Science and Technology, P.O. Box 447, Arusha, Tanzania \\ ${ }^{2}$ Department of Mathematics, Department of Mathematics, Mkwawa University College of Education, P.O. Box 2513, \\ Iringa, Tanzania \\ Correspondence should be addressed to Theresia Shirima Sabini; theresiasabini88@gmail.com
}

Received 8 August 2019; Accepted 26 February 2020; Published 30 March 2020

Academic Editor: Vladimir V. Mityushev

Copyright ( $\odot 2020$ Theresia Shirima Sabini et al. This is an open access article distributed under the Creative Commons Attribution License, which permits unrestricted use, distribution, and reproduction in any medium, provided the original work is properly cited.

\begin{abstract}
Bovine tuberculosis (bTB) is a bacterial and zoonotic disease which is transmitted through consumption of unpasteurized milk and uncooked meat and inhalation of aerosols. In this paper, a deterministic mathematical model is formulated to study the transmission dynamics of bTB in humans and animals. The basic reproduction number $R_{0}$ is computed to determine the behavior of the disease. Stability analysis shows that there is a possibility for disease-free equilibrium and endemic equilibrium to coexist when $R_{0}=1$. To determine parameters which drive the dynamics of bTB, we performed sensitivity analysis. The analysis shows that the rate at which dairy products are produced, the rate of transmission of bTB from animal to animal, and the rates at which human acquires bTB from infectious dairy products and animals drive the transmission of bTB. However, the disease decreases as the rate of consumption of dairy products decreases. To control bTB, education campaign, inspection of dairy products, treatment of infected humans, and quarantine of infected animals are recommended.
\end{abstract}

\section{Introduction}

Tuberculosis (TB) is a global health problem which is among the top ten diseases which are leading in causing death for many people [1]. World Health Organization (WHO) has conducted several meetings to discuss strategies on how to end TB but the disease is still claiming lives. According to WHO report of 2018, TB kills 1.3 million people among HIV negative, and there was an addition of 300,000 people with HIV positive who died with TB. It is estimated that annually 10 million people get ill with TB of which 5.8 million are men, 3.2 million are women, and 1.0 million are children [1]. Africa is reported to have highest number of cases, followed by India, China, and Indonesia with percentage $72 \%, 27 \%$, $9 \%$, and $8 \%$, respectively [1]. Although TB is a worldwide health problem, other forms of TB such as zoonotic tuberculosis are still neglected.

Bovine tuberculosis (bTB) is a bacterial and zoonotic disease, which was originally transmitted to cattle from wild animals especially buffalo and badger, and then spread to other domestic animals like cows, goats, pigs, horses, and sheep [2]. The disease has a great negative economic impact due to slaughtering of infectious animals when they acquire bTB [3]. Also, bTB causes human health problems which sometimes cost lives. It can lead to loss of self-employment for some workers, especially those who depend on livestock keeping as their main source of income [4]. Bovine tuberculosis is transmitted to humans through three main ways, which are, consumption of unpasteurized milk, eating uncooked meat, and inhalation of aerosols $[5,6]$. In animals, bTB is transmitted when there is close interaction between uninfected animal and infectious animals [7] and consumption of infectious milk especially during breastfeeding and inhalation of aerosols [8].

It is estimated that about 147,000 new cases of bTB in humans were reported, whereby 12,000 people die annually due this disease [2]. In Tanzania, the disease prevalence varies from region to region depending on the number of livestock in a particular place and it ranges from $0.2 \%-13.3 \%$ $[6,9]$. The problem of not having surveillance data makes the estimation to be poor and difficult [3]. Places where bTB exist, includes Northern Tanzania (Arusha, Kilimanjaro, and 
Manyara), dairy farms in Kibaha, and some areas in Morogoro districts $[3,6]$.

Early diagnosis of bTB helps to know the dynamics of the disease and identify ways of controlling the transmission factors before it becomes endemic. Various tools and methods are used to diagnose bTB and some of them include Polymerase chain reaction (PCR) and gene sequencing of culture isolate which is an expensive tool $[10,11]$, post mortem examination which focused on lymph nodes [12], and single itradermal comparative cervical test (SICCT) [13]. From external examination it shows that there is high possibility of carcasses from slaughtered cattle to contain bTB pathogens [14].

Various organizations such as WHO, OIE, and FAO joined together to fight against transmission of bTB in order to eradicate the disease [2]. Though different approaches such as "one health approach, together we can save lives, and secure livelihoods" are used, bTB is still a problem especially in some African countries. Though spread of bTB has been controlled by treating livestock using various medicines, such as pyrazinamide medicine, unfortunately, bTB is still resistant to pyrazinamide medicine because it is used to treat patients with pulmonary TB [2]. Many studies have ignored the role of dairy products in the spread of bTB. This study aims to find the parameters which drive the dynamics of bTB and explore the role played by dairy products in the transmission dynamics of bTB.

Studies such as Agusto et al. [15], Liu et al. [16], Mathews et al. [11], and Phepa [17] developed mathematical models to study the transmission dynamics of bTB and its control strategies. Most of these studies did not consider the impacts of dairy products in the transmission of bTB although the products are among the factors which drive the transmission of bTB since they are consumed by large number of people worldwide $[18,19]$. These products are among the factors which lead in the spread of bTB as some of the findings reported the products to contain Ramos et al. [20]. This study uses a mathematical model to study transmission dynamics of bTB in human beings and livestock by considering dairy products as a risk factor for the transmission of bTB.

This work is organized as follows: materials and methods are presented in the Section 2 followed by model analysis, sensitivity analysis, and numerical simulation, and it is concluded with conclusion and recommendation.

\section{Materials and Methods}

2.1. Model Formulation. The model is formulated by modifying the tuberculosis model for human and cows in Urumqi, Xinjiang, China which was developed by Liu et al. [16]. The model divides cow population into three groups: Susceptible $S_{c}$, Infected $I_{c}$, and Quarantine $Q_{c}$ (SIQ) and human population into four groups: Susceptible $S_{h}$, Exposed $E_{h}$, Infected $I_{h}$, and Recovery $R_{h}$ (SEIR).

Our current model includes animal and human populations. Human population is divided into susceptible $S_{H}$, exposed $E_{H}$, and infected $I_{H}(\mathrm{SEI})$ and animal population is divided into susceptible $S_{a}$, exposed $E_{a}$, and infected $I_{a}$ compartments. The variable $D$ represents diary products which are produced by infected animals. The proposed model does not include recovery class because it is assumed that there is no natural recovery [21].

Susceptible humans are recruited through birth and migration at a rate $\Lambda_{H}$, and they acquire bovine tuberculosis latent infection following contacts with infectious human and animals and after consuming dairy products from infectious animals at a rate

$$
\lambda_{H}=\frac{\left(\beta_{1} I_{H}+\beta_{2} I_{a}+\beta_{3} D\right)}{N_{H}} .
$$

Exposed compartment $E_{H}$ increases the following latent infection of susceptible humans $S_{H}$ at a rate $\lambda_{H}$ and it decreases due to progression to infectious stage at a rate $\gamma_{H}$. Infectious humans $I_{H}$ increase at a rate $\gamma_{H}$ and diminish due to disease-induced mortality at a rate $\alpha_{H}$. All human compartments suffer natural mortality at a rate $\mu_{H}$.

Susceptible animals $S_{a}$ are recruited through birth and migration at a rate $\Lambda_{a}$ and acquire bovine tuberculosis latent infection following contacts with infectious humans and animals and after consuming dairy products at a rate

$$
\lambda_{a}=\frac{\beta_{4} I_{H}+\beta_{5} I_{a}+\beta_{6} D}{N_{a}} .
$$

Exposed animals $E_{a}$ increase following latent infection of susceptible animals $S_{a}$ at a rate $\lambda_{a}$. However, they decrease due to progression to the infectious stage at a rate $\gamma_{a}$. Infectious animals $I_{a}$ increase at a rate $\gamma_{a}$ and diminish due to disease-induced mortality at a rate $\alpha_{a}$. All animal compartments suffer natural mortality at a rate $\mu_{a}$.

Dairy products are produced by infectious animals at a rate $\rho$ and leak out at a rate $\omega$, and susceptible humans consumed dairy products at a rate $\beta_{3}$ and susceptible animals at a rate $\beta_{6}$.

In the model we assume that all humans and animals are susceptible to the disease. Susceptible human $S_{H}$ contacts bTB when they consume dairy products $D$ such as milk and meat from infected animals; when they inhale aerosols from infected animals and human and have direct contact with dairy product from infected animals through scratches [5]. Susceptible animal acquires infection when they interact with infectious animals and humans, through breastfeeding from infectious animals and inhalation of aerosols. There is constant natural death to both animals and human beings. There is no natural recovery for infected individuals.

Figure 1 demonstrates the interaction of state variables; Tables 1 and 2 describe state variables and parameters, respectively.

2.2. Model Equations. From Figure 1, we have the following system of differential equations:

$$
\begin{aligned}
& \frac{\mathrm{d} S_{H}}{\mathrm{~d} t}=\Lambda_{H}-\left(\frac{\beta_{1} I_{H}+\beta_{2} I_{a}+\beta_{3} D}{N_{H}}\right) S_{H}-\mu_{H} S_{H}, \\
& \frac{\mathrm{d} E_{H}}{\mathrm{~d} t}=\left(\frac{\beta_{1} I_{H}+\beta_{2} I_{a}+\beta_{3} D}{N_{H}}\right) S_{H}-\left(\gamma_{H}+\mu_{H}\right) E_{H},
\end{aligned}
$$




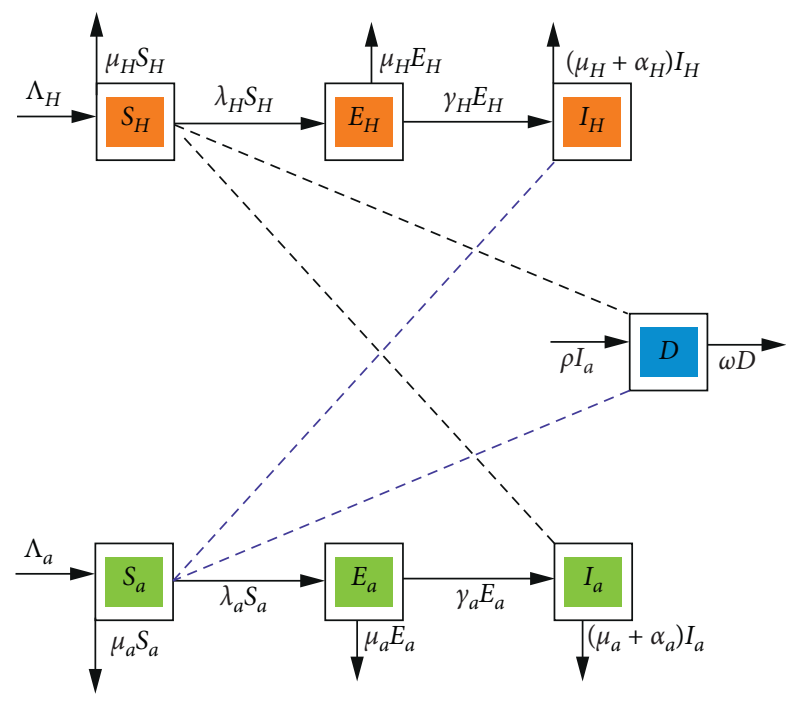

Figure 1: Model flow diagram.

TABLE 1: Model variables description.

\begin{tabular}{lc}
\hline Symbol & Description \\
\hline$S_{H}(t)$ & Number of susceptible human at time $t$ \\
$S_{a}(t)$ & Number of susceptible animal at time $t$ \\
$E_{H}(t)$ & Number of exposed/latent human beings at time $t$ \\
$E_{a}(t)$ & Number of exposed animals at time $t$ \\
$I_{H}(t)$ & Number of infected human at time $t$ \\
$I_{a}(t)$ & Number of infected animals at time $t$ \\
$D(t)$ & Dairy products at time $t$
\end{tabular}

TABle 2: Parameters' descriptions.

\begin{tabular}{lc}
\hline Parameter & Descriptions \\
\hline$\Lambda_{H}$ & Human recruitment rate \\
$\mu_{H}$ & Human natural death \\
$\gamma_{H}$ & Progression rate from $E_{H}$ to $I_{H}$ \\
$\alpha_{H}$ & Human death rate due to disease induced \\
$\beta_{1}, \beta_{2}, \beta_{3}$ & Humans infection rate from $I_{H}, I_{a}$, and $D$, respectively \\
$\Lambda_{a}$ & Animal recruitment rate \\
$\mu_{a}$ & Animal natural death rate \\
$\gamma_{a}$ & Progression rate from $E_{a}$ to $I_{a}$ \\
$\alpha_{a}$ & Animals disease induced mortality \\
$\rho$ & Dairy products production rate \\
$\omega$ & Rate of decaying for unconsumed dairy products \\
$\beta_{4}, \beta_{5}, \beta_{6}$ & Animals infection rate from $I_{H}, I_{a}$, and $D$, respectively \\
\hline
\end{tabular}

$$
\begin{aligned}
& \frac{\mathrm{d} I_{H}}{\mathrm{~d} t}=\gamma_{H} E_{H}-\left(\mu_{H}+\alpha_{H}\right) I_{H} \\
& \frac{\mathrm{d} S_{a}}{\mathrm{~d} t}=\Lambda_{a}-\left(\frac{\beta_{4} I_{H}+\beta_{5} I_{a}+\beta_{6} D}{N_{a}}\right) S_{a}-\mu_{a} S_{a} \\
& \frac{\mathrm{d} E_{a}}{\mathrm{~d} t}=\left(\frac{\beta_{4} I_{H}+\beta_{5} I_{a}+\beta_{6} D}{N_{a}}\right) S_{a}-\left(\gamma_{a}+\mu_{a}\right) E_{a} \\
& \frac{\mathrm{d} I_{a}}{\mathrm{~d} t}=\gamma_{a} E_{a}-\left(\mu_{a}+\alpha_{a}\right) I_{a}
\end{aligned}
$$

$$
\frac{\mathrm{d} D}{\mathrm{~d} t}=\rho I_{a}-\omega D,
$$

subject to their initial conditions: $S_{H}(0)=0, E_{H}(0)=0, I_{H}(0)$ $=0, S_{a}(0)=0, E_{a}(0)=0, I_{a}(0)=0$, and $D(0)=0$.

\section{Model Analysis}

To determine whether the model is mathematically and epidemiologically meaningful, we find invariant region and test positivity of solutions. The model is biologically and mathematically meaningful, when its solutions are positive and bounded.

3.1. Invariant Region. Invariant region shows the feasibility of the model solutions. To study the feasibility of the model solutions, we denote humans and animal populations by $N_{H}$ and $N_{a}$, respectively.

Beginning with human population, we have

$$
\begin{aligned}
N_{H} & =S_{H}+E_{H}+I_{H}, \\
\frac{\mathrm{d} N_{H}}{\mathrm{~d} t} & =\Lambda_{H}-\mu_{H} N_{H}-\alpha_{H} I_{H} .
\end{aligned}
$$

Solving (4), we obtain

$$
N_{H}(0) \leq \frac{\Lambda_{H}}{\mu_{H}}+\left(N_{H}(0)-\frac{\Lambda_{H}}{\mu_{H}}\right) e^{-\mu_{H} t} .
$$

Analysis of $N_{H}$ consider two cases

$$
\text { when } N_{H}(0)>\frac{\Lambda_{H}}{\mu_{H}} \text { and when } N_{H}(0)<\frac{\Lambda_{H}}{\mu_{H}} \text {. }
$$

For all two cases, we have

$$
\begin{aligned}
& N_{H}(t) \leq \frac{\Lambda_{H}}{\mu_{H}} \leq \frac{\Lambda_{H}}{\mu_{H}}+\left(N_{H}(0)-\frac{\Lambda_{H}}{\mu_{H}}\right) e^{-\mu_{H} t}, \\
& N_{H}(t) \leq \frac{\Lambda_{H}}{\mu_{H}}+\left(N_{H}(0)-\frac{\Lambda_{H}}{\mu_{H}}\right) e^{-\mu_{H} t} \leq \frac{\Lambda_{H}}{\mu_{H}} .
\end{aligned}
$$

As $t \longrightarrow \infty$,

$$
0 \leq N_{H} \leq \frac{\Lambda_{H}}{\mu_{H}} .
$$

When we apply the same procedure for animals population, as $t \longrightarrow \infty$,

$$
0 \leq N_{a} \leq \frac{\Lambda_{a}}{\mu_{a}}
$$

Since $N_{a} \leq\left(\Lambda_{a} / \mu_{a}\right)$, then $I_{a} \leq\left(\Lambda_{a} / \mu_{a}\right)$.

For the case of dairy products, when $t \longrightarrow \infty$ we have

$$
D(t) \leq \frac{\Lambda_{a}}{\mu_{a}}\left(\frac{\rho}{\omega}\right) .
$$

Therefore, we can see that model (3) is a positive invariant in the region 


$$
Z=\left\{\left(S_{H}, E_{H}, I_{H}, S_{a}, E_{a}, I a, D\right) \in R_{+}^{7}: 0 \leq N_{H} \leq \frac{\Lambda_{H}}{\mu_{H}} ; 0 \leq N_{a} \leq \frac{\Lambda_{a}}{\mu_{a}} ; 0 \leq D \leq \frac{\Lambda a}{\mu_{a}}\left(\frac{\rho}{\omega}\right)\right\}
$$

\subsubsection{Positivity of Solution}

Theorem 1. Let the initial variable of model (3) be $S_{H}(0)>0$, $E_{H}(0)>0, I_{H}(0)>0, S_{a}(0)>0, E_{a}(0)>0, I_{a}(0)>0$, and $D>0$; then, the solutions $S_{H}>0, E_{H}>0, I_{H}>0, S_{a}>0, E_{a}>0, I_{a}$, and $D>0$ are positive $\forall t>0$.

Proof:. Let us consider equation (3a) of the model system (3), which is

$$
\begin{aligned}
& \frac{\mathrm{d} S_{H}}{\mathrm{~d} t}=\Lambda_{H}-\left(\frac{\beta_{1} I_{H}+\beta_{2} I_{a}+\beta_{3} D}{N_{H}}\right) S_{H}-\mu_{H} S_{H}, \\
& \frac{\mathrm{d} S_{H}}{\mathrm{~d} t} \geq-\left(\frac{\beta_{1} I_{H}+\beta_{2} I_{a}+\beta_{3} D}{N_{H}}\right) S_{H}-\mu_{H} S_{H} .
\end{aligned}
$$

By separating variables (12) and integrating, we obtain

$$
\begin{aligned}
& \frac{\mathrm{d} S_{H}}{S_{H}} \geq-\left(\frac{\beta_{1} I_{H}+\beta_{2} I_{a}+\beta_{3} D}{N_{H}}+\mu_{H}\right) \mathrm{d} t, \\
& \int \frac{\mathrm{d} S_{H}}{S_{H}} \geq-\int_{0}^{t}\left(\frac{\beta_{1} I_{H}(s)+\beta_{2} I_{a}(s)+\beta_{3} D(s)}{N_{H}(s)}+\mu_{H}\right) \mathrm{d} s, \\
& \ln S_{H} \geq-\int_{0}^{t}\left(\frac{\beta_{1} I_{H}(s)+\beta_{2} I_{a}(s)+\beta_{3} D(s)}{N_{H}(s)}+\mu_{H}\right) \mathrm{d} s+C, \\
& S_{H} \geq \mathrm{Ce} \int_{0}^{t}-\left(\frac{\beta_{1} I_{H}(s)+\beta_{2} I_{a}(s)+\beta_{3} D(s)}{N_{H}(s)}+\mu_{H}\right) \mathrm{d} s .
\end{aligned}
$$

At initial condition, we obtain

$$
S_{H} \geq S_{H}(0) e^{\int_{0}^{t}-\left(\frac{\beta_{1} I_{H}(s)+\beta_{2} I_{a}(s)+\beta_{3} D(s)}{N_{H}(s)}+\mu_{H}\right) d s} .
$$

Following the same approach for the remaining variables (3b)-(3g) of the model system (3), we obtain

$$
\begin{aligned}
E_{H} & \geq E_{H}(0) e^{-\left(\gamma_{H}+\mu_{H}\right) t}, \\
I_{H} & \geq \mathrm{Ce}^{\left(\mu_{H}+\alpha_{H}\right) t}, \\
S_{a} & \geq S_{a}(0) e^{\int_{0}^{t}-\left(\frac{\beta_{4} I_{H}(s)+\beta_{5} I_{a}(s)+\beta_{6} D(s)}{N_{a}(s)}+\mu_{a}\right) d s}, \\
E_{a} & \geq E_{a}(0) e^{-\left(\gamma_{a}+\mu_{a}\right) t}, \\
I_{a} & \geq I_{a}(0) e^{-\left(\mu_{a}+\alpha_{a}\right) t}, \\
D & \geq D(0) e^{-\omega t} .
\end{aligned}
$$

\section{Since}

$$
S_{H}>0, E_{H}>0, I_{H}>0, S_{a}>0, E_{a}>0, I_{a}>0 \text { and } D>0 \text {, }
$$

then the model solutions are positive $\forall t>0$.

Model (3) is mathematically and epidemiologically meaningful; therefore, we can consider the flow generated by the model for analysis.

3.2. Disease-Free Equilibrium (DFE). The disease-free equilibrium point is the state when there is no disease in the population. When there is no bTB in human and animal populations, the disease-free equilibrium is given by

$$
\mathrm{DF}^{0}=\left(S_{H}, E_{H}, I_{H}, S_{a}, E_{a}, I_{a}, D\right)=\left(\frac{\Lambda_{H}}{\mu_{H}}, 0,0, \frac{\Lambda_{a}}{\mu_{a}}, 0,0,0\right)
$$

3.2.1. The Basic Reproduction Number $R_{0}$. The basic reproduction number refers to the average number of new cases that a single infectious individual causes when introduced into an entirely susceptible population [22]. It determines whether the disease persists or clears out in the population. When the basic reproduction number $R_{0}<1$, the disease clears out in the population and it persists if the basic reproduction number $R_{0}>1$. This is because when an infectious individual is introduced into an entirely susceptible population, he/she infects more than one individual, hence the disease persists $[23,24]$.

To compute the basic reproduction number $R_{0}$, we use the next generation matrix method where new infections and transfer terms are considered $[22,24]$. If bTB new infectious and transfer terms are denoted by $F_{i}$ and $V_{i}$, respectively, then the basic reproduction number $R_{0}$ is given as the maximum eigenvalue. That is,

$$
R_{0}=\rho\left(\mathrm{FV}^{-1}\right)
$$

where

$$
F=\frac{\partial F_{i}}{\partial X_{j}}\left(\mathrm{DF}^{0}\right) \text { and } V=\frac{\partial V_{i}}{\partial X_{j}}\left(D F^{0}\right)
$$

From the model system (3), the basic reproduction number $R_{0}$ is given by 


$$
\begin{aligned}
R_{0}= & \frac{\left(\beta_{1} \gamma_{H} /\left(\mu_{H}+\gamma_{H}\right)\left(\mu_{H}+\alpha_{H}\right)+\gamma_{a}\left(\omega \beta_{5}+\rho \beta_{6}\right) /\left(\gamma_{a}+\mu_{a}\right)\left(\mu_{a}+\alpha_{a}\right) \omega\right)}{2} \\
& +\sqrt{\frac{\left(\beta_{1} \gamma_{H} /\left(\mu_{H}+\gamma_{H}\right)\left(\mu_{H}+\alpha_{H}\right)-\gamma_{a}\left(\omega \beta_{5}+\rho \beta_{6}\right) /\left(\gamma_{a}+\mu_{a}\right)\left(\mu_{a}+\alpha_{a}\right) \omega\right)^{2}+4 c e}{4}}, \\
\text { where ce }= & \frac{\gamma_{H} \gamma_{a}\left(\omega \beta_{2}+\rho \beta_{3}\right)}{\left(\gamma_{H}+\mu_{H}\right)\left(\alpha_{H}+\mu_{H}\right)\left(\gamma_{a}+\mu_{a}\right) \omega} .
\end{aligned}
$$

The terms $1 /\left(\gamma_{H}+\mu_{H}\right)$ and $1 /\left(\gamma_{a}+\mu_{a}\right)$ in (20) are the average periods an individual human and animal spends in their corresponding exposed classes, $1 /\left(\alpha_{H}+\mu_{H}\right)$ and $1 /\left(\alpha_{a}+\mu_{a}\right)$ are the average periods an infectious human and animal spend in their infectious classes, $\beta_{1} \gamma_{H} /\left(\gamma_{H}+\mu_{H}\right)\left(\alpha_{H}+\mu_{H}\right)$ and $\beta_{4} \gamma_{H} /\left(\gamma_{H}+\mu_{H}\right)\left(\alpha_{H}+\mu_{H}\right)$ are the proportions of infected humans that develop bTB and move from exposed class to infectious class after contacting infectious humans and animals, respectively, $\left(\omega \beta_{5}+\rho \beta_{6}\right) \gamma_{a} /\left(\gamma_{a}+\mu_{a}\right)\left(\alpha_{a}+\mu_{a}\right) \omega$ is the sum of proportions of infected animals that progress from exposed class to infectious class after coming into contact with infectious animals and after consuming infectious dairy products, and $\gamma_{H} \gamma_{a}\left(\omega \beta_{2}+\rho \beta_{3}\right) /\left(\gamma_{H}+\mu_{H}\right)\left(\alpha_{H}+\mu_{H}\right)\left(\gamma_{a}+\mu_{a}\right)\left(\alpha_{a}+\mu_{a}\right) \omega$ is the sum of proportions of infected humans who develop bTB by contacting infectious animals and after consuming infectious dairy products.

3.3. Sensitivity Analysis of Basic Reproduction Number $\left(R_{0}\right)$. Sensitivity analysis of $R_{0}$ helps to understand the effect of each parameter on the model output and their influence in the spread of disease in the population $[25,26]$. We perform sensitivity analysis of $R_{0}$ by using normalized forward sensitivity analysis index as used by Chitnis et al. [27] and Silva and Torres [26]. A normalized forward sensitivity index of variable $\beta$ with respect to basic reproduction number $R_{0}$ is defined as
Using estimated parameters and from related literature, sensitivity index of each parameter with respect to basic reproduction number $R_{0}$ is computed and summarized in Table 3.

Sensitivity analysis shows that human infection rates due to consumption of dairy products $\beta_{3}$ and contact rate with infected animals $\beta_{2}$, animal infection rates due to contact with infectious animals $\beta_{5}$, and consumption of diary product $\beta_{6}$ drive the dynamics of bTB. Generally, the most sensitive parameter is the rate of producing dairy products $\rho$. As dairy products increases by $10 \%$, the basic reproduction number $R_{0}$ increases by $3.89 \%$. However, when animal mortality rate due to disease $\alpha_{a}$, natural death rate for animals $\mu_{a}$, human disease-induced death rate $\alpha_{H}$, natural death rate for humans $\mu_{H}$, and dairy products decaying rate $\omega$ increases, and the basic reproduction number $R_{0}$ decrease consequently.

3.4. Stability Analysis for Disease-Free Equilibrium (DFE). To determine local stability of disease-free equilibrium, we use the linearization method where trace and determinant are used. Disease-free equilibrium is said to be locally asymptotically stable if the eigenvalues of matrix (22) are negative or have a negative real part. Linearization of the model system (3) gives the matrix

$$
J=\left[\begin{array}{ccccccc}
-\mu_{H} & 0 & -\beta_{1} & 0 & 0 & -\beta_{2} & -\beta_{3} \\
0 & -\mu_{H}-\gamma_{H} & \beta_{1} & 0 & 0 & \beta_{2} & \beta_{3} \\
0 & \gamma_{H} & -\mu_{H}-\alpha_{H} & 0 & 0 & 0 & 0 \\
0 & 0 & -\beta_{4} & -\mu_{a} & 0 & -\beta_{5} & -\beta_{6} \\
0 & 0 & \beta_{4} & 0 & -\mu_{a}-\gamma_{a} & \beta_{5} & \beta_{6} \\
0 & 0 & 0 & 0 & \gamma_{a} & -\mu_{a}-\alpha_{a} & 0 \\
0 & 0 & 0 & 0 & 0 & \rho & -\omega
\end{array}\right] .
$$


TABle 3: Sensitivity indices for $R_{0}$.

\begin{tabular}{lc}
\hline Parameter & Index value \\
\hline$\beta_{1}$ & 0.0271 \\
$\beta_{2}$ & 0.0530 \\
$\beta_{3}$ & 0.1177 \\
$\beta_{4}$ & 0.1708 \\
$\beta_{5}$ & 0.3601 \\
$\beta_{6}$ & 0.2713 \\
$\gamma_{H}$ & 0.0892 \\
$\mu_{H}$ & -0.2728 \\
$\alpha_{H}$ & -0.0144 \\
$\gamma_{a}$ & 0.1671 \\
$\alpha_{a}$ & -0.5793 \\
$\mu_{a}$ & -0.3898 \\
$\rho$ & 0.3890 \\
$\omega$ & -0.3890 \\
\hline
\end{tabular}

Matrix (22) has negative eigenvalues $-\mu_{H}$ and $-\mu_{a}$. Matrix (22) now reduces to

$$
K=\left[\begin{array}{ccccc}
-\mu_{H}-\gamma_{H} & \beta_{1} & 0 & \beta_{2} & \beta_{3} \\
\gamma_{H} & -\mu_{H}-\alpha_{H} & 0 & 0 & 0 \\
0 & \beta_{4} & -\mu_{a}-\gamma_{a} & \beta_{5} & \beta_{6} \\
0 & 0 & \gamma_{a} & -\mu_{a}-\alpha_{a} & 0 \\
0 & 0 & 0 & \rho & -\omega
\end{array}\right] .
$$

We analyze matrix $K$ by using trace tr and determinant det. Disease-free equilibrium is locally stable if trace is negative $\operatorname{tr}(K)<0$ and determinant is positive $\operatorname{det}(K)>0$. From (23), trace of the matrix $K$ is given by

$$
\begin{aligned}
\operatorname{tr}(K)= & -\left(\left(\mu_{H}+\gamma_{H}\right)+\left(\mu_{H}+\gamma_{H}\right)+\left(\mu_{a}+\gamma_{a}\right)\right. \\
& \left.+\left(\mu_{a}+\alpha_{a}\right)+\omega\right), \operatorname{tr}(K)<0,
\end{aligned}
$$

and $\operatorname{det}(K)$ is given by

$$
\begin{aligned}
\operatorname{det}(K)= & \left(\gamma_{H}+\mu_{H}\right)\left(\alpha_{H}+\mu_{H}\right) \omega \beta_{5} \gamma_{a}+\left(\gamma_{H}+\mu_{H}\right) \\
& \cdot\left(\mu_{H}+\alpha_{H}\right) \rho \beta_{6} \gamma_{a}+\left(\gamma_{a}+\mu_{a}\right)\left(\alpha_{a}+\mu_{a}\right) \omega \beta_{1} \gamma_{H} \\
& +\omega \beta_{2} \beta_{4} \gamma_{H} \gamma_{a}+\rho \beta_{3} \beta_{4} \gamma_{H} \gamma_{a}-\rho \beta_{1} \beta_{6} \gamma_{H} \gamma_{a} \\
& -\left(\gamma_{H}+\mu_{H}\right)\left(\alpha_{H}+\mu_{H}\right)\left(\gamma_{a}+\mu_{a}\right)\left(\alpha_{a}+\mu_{a}\right) \omega \\
& -\omega \beta_{1} \beta_{5} \gamma_{H} \gamma_{a}
\end{aligned}
$$

$$
\operatorname{det}(K)>0 \text { if }
$$

$$
\begin{gathered}
\frac{\beta_{1} \gamma_{H}}{\left(\mu_{H}+\gamma_{H}\right)\left(\mu_{H}+\alpha_{H}\right)}+\frac{\gamma_{a}\left(\omega \beta_{5}+\rho \beta_{6}\right)}{\omega\left(\gamma_{a}+\mu_{a}\right)\left(\mu_{a}+\alpha_{a}\right)} \\
+\frac{\beta_{4} \gamma_{H}\left(\omega \beta_{2} \gamma_{a}+\rho \beta_{3} \gamma_{a}\right)}{\omega\left(\gamma_{H}+\mu_{H}\right)\left(\alpha_{H}+\mu_{H}\right)\left(\gamma_{a}+\mu_{a}\right)\left(\alpha_{a}+\mu_{a}\right)} \\
-\frac{\gamma_{H} \gamma_{a} \beta_{1}\left(\omega \beta_{5}+\rho \beta_{6}\right)}{\omega\left(\gamma_{H}+\mu_{H}\right)\left(\alpha_{H}+\mu_{H}\right)\left(\gamma_{a}+\mu_{a}\right)\left(\alpha_{a}+\mu_{a}\right)}>1 .
\end{gathered}
$$

Theorem 1. The disease-free equilibrium is locally asymptotically stable if condition (26) holds and $R_{0}<1$.

However, the disease-free equilibrium may not be globally asymptotically stable due to the possibility of model (3) to undergo backward bifurcation when $R_{0}=1$. We analyze backward bifurcation in Section 3.5.

3.5. Bifurcation Analysis. To determine the possibility of model (3) to undergo backward bifurcation, we rename the state variables $S_{H}, E_{H}, I_{H}, S_{a}, E_{a}, I_{a}$, and $D$ to be $x_{1}, x_{2}, x_{3}, x_{4}$, $x_{5}, x_{6}$, and $x_{7}$, respectively, where $N_{H}=x_{1}+x_{2}+x_{3}$ and $N_{a}=x_{4}+x_{5}+x_{7}$. By introducing the vector notations $X=\left(x_{1}, x_{2}, x_{3}, x_{4}, x_{5}, x_{6}, x_{7}\right)^{T}$, the model system (3) is now written as $(\mathrm{d} X / \mathrm{d} t)=F(X)$, where $F(X)=\left(f_{1}, f_{2}, f_{3}, f_{4}\right.$, $\left.f_{5}, f_{6}, f_{7}\right)^{T}$. The model system (3) is then rewritten as follows:

$$
\begin{aligned}
& \frac{\mathrm{d} x_{1}}{\mathrm{~d} t}=f_{1}=\Lambda_{H}-\left(\frac{\beta_{1} x_{3}+\beta_{2} x_{6}+\beta_{3} x_{7}}{x_{1}+x_{2}+x_{3}}\right) x_{1}-\mu_{H} x_{1}, \\
& \frac{\mathrm{d} x_{2}}{\mathrm{~d} t}=f_{2}=\left(\frac{\beta_{1} x_{3}+\beta_{2} x_{6}+\beta_{3} x_{7}}{x_{1}+x_{2}+x_{3}}\right) x_{1}-\left(\gamma_{H}+\mu_{H}\right) x_{2}, \\
& \frac{\mathrm{d} x_{3}}{\mathrm{~d} t}=f_{3}=\gamma_{H} x_{2}-\left(\mu_{H}+\alpha_{H}\right) x_{3}, \\
& \frac{\mathrm{d} x_{4}}{\mathrm{~d} t}=f_{4}=\Lambda_{a}-\left(\frac{\beta_{4} x_{3}+\beta_{5} x_{6}+\beta_{6} x_{7}}{x_{4}+x_{5}+x_{6}}\right) x_{4}-\mu_{a} x_{4}, \\
& \frac{\mathrm{d} x_{5}}{\mathrm{~d} t}=f_{5}=\left(\frac{\beta_{4} x_{3}+\beta_{5} x_{6}+\beta_{6} x_{7}}{x_{4}+x_{5}+x_{6}}\right) x_{4}-\left(\gamma_{a}+\mu_{a}\right) x_{5}, \\
& \frac{\mathrm{d} x_{6}}{\mathrm{~d} t}=f_{6}=\gamma_{a} x_{5}-\left(\mu_{a}+\alpha_{a}\right) x_{6}, \\
& \frac{\mathrm{d} x_{7}}{\mathrm{~d} t}=f_{7}=\rho x_{6}-\mu_{D} x_{7} .
\end{aligned}
$$

The Jacobian of system (27) at disease-free equilibrium is given by

$$
J=\left[\begin{array}{ccccccc}
-\mu_{H} & 0 & -\beta_{1} & 0 & 0 & -\beta_{2} & -\beta_{3} \\
0 & -\mu_{H}-\gamma_{H} & \beta_{1} & 0 & 0 & \beta_{2} & \beta_{3} \\
0 & \gamma_{H} & -\mu_{H}-\alpha_{H} & 0 & 0 & 0 & 0 \\
0 & 0 & -\beta_{4} & -\mu_{a} & 0 & -\beta_{5} & -\beta_{6} \\
0 & 0 & \beta_{4} & 0 & -\mu_{a}-\gamma_{a} & \beta_{5} & \beta_{6} \\
0 & 0 & 0 & 0 & \gamma_{a} & -\mu_{a}-\alpha_{a} & 0 \\
0 & 0 & 0 & 0 & 0 & \rho & -\omega
\end{array}\right] .
$$

To determine whether system (27) undergoes backward bifurcation at $R_{0}=1$, we adopt Theorem 1 in Castillo-Chavez and Song [28], and it is restated as follows. 
Theorem 2. Consider the following general system of ordinary differential equations with a parameter $\beta^{*} .(d x / d t)=f\left(x, \beta^{*}\right), f: \mathfrak{R} \times \mathfrak{R}^{n} \mapsto \mathfrak{R}^{n}$, and $f \in C^{2}\left(\mathfrak{R}^{n} \times\right.$ $\mathfrak{R}$ ), where 0 is an equilibrium point of the system (that is, $f(0$, $\left.\beta^{*}\right) \equiv 0 \forall \beta^{*}$ and

(1) $A=D_{x} f(0,0)=\left(\partial f_{i} / \partial x_{j}\right)(0,0)$ is a linearization matrix of the system around the equilibrium 0 with $\beta^{*}$ at 0 .

(2) Zero is a simple eigenvalue of $A$ and all other eigenvalues of $A$ have negative real parts.

(3) Matrix A has a right eigenvectors $w$ and left eigenvectors $v$ corresponding to the zero eigenvalues.

Let $f_{k}$ be the $k^{\text {th }}$ component of $f$ and

$$
\begin{aligned}
& a=\sum_{k, i, j=1}^{n} v_{k} w_{i} w_{j} \frac{\partial^{2} f_{k}}{\partial x_{i} \partial x_{j}}(0,0), \\
& b==\sum_{k, i=1}^{n} v_{k} w_{i} \frac{\partial^{2} f_{k}}{\partial x_{i} \partial \beta^{*}}(0,0) .
\end{aligned}
$$

Then, the local dynamics of the system around the equilibrium point is totally determined by the signs of $a$ and $b$. Particularly, if $a>0$ and $b>0$ then a backward bifurcation occurs at $\beta^{*}=0$.

The local dynamics at (27) around 0 are totally determined by signs of $a$ and $b$.

(i) $a>0$ and $b>0$. When $\beta^{*}<0$ with $\left|\beta^{*}\right|<<1,0$ is locally asymptotically stable, and there exists a positive unstable equilibrium; when $0<\beta^{*}<<1,0$ is unstable and there exists a negative and locally asymptotically stable equilibrium.

(ii) $a<0$ and $b<0$. When $\beta^{*}<0$ with $\left|\beta^{*}\right|<<1,0$ is unstable; when $0<\beta^{*}<<1,0$ is locally asymptotically stable, and there exists a positive unstable equilibrium.

(iii) $a>0$ and $b<0$. When $\beta^{*}<0$ with $\left|\beta^{*}\right|<<1,0$ is unstable, and there exists a locally asymptotically stable negative equilibrium; when $0<\beta^{*}<<1,0$ is stable, and a positive unstable equilibrium appears.

(iv) $a<0$ and $b>0$. When $\beta^{*}$ changes from negative to positive, 0 changes its stability from stable to unstable. Correspondingly, a negative unstable equilibrium becomes positive and locally asymptotically stable.

Let $\beta_{3}=\beta^{*}$ be a bifurcation parameter when $R_{0}=1$. Now, solving for $\beta_{3}=\beta^{*}$ when $R_{0}=1$, we obtain

$$
\begin{aligned}
\beta_{3}= & \beta^{*}=M\left(2-\frac{\beta_{4} \gamma_{H}}{\left(\gamma_{H}+\mu_{H}\right)\left(\alpha_{H}+\mu_{H}\right)}-\frac{\gamma_{a}\left(\rho \beta_{6}+\omega \beta_{2}\right)}{\left(\gamma_{a}+\mu_{a}\right)\left(\alpha_{a}+\mu_{a}\right)}\right)^{2} \\
& +M\left(\frac{\beta_{1} \gamma_{H}}{\left(\gamma_{H}+\mu_{H}\right)\left(\alpha_{H}+\mu_{H}\right)}-\frac{\gamma_{a}\left(\rho \beta_{6}+\omega \beta_{2}\right)}{\left(\gamma_{a}+\mu_{a}\right)\left(\alpha_{a}+\mu_{a}\right)}\right)^{2} .
\end{aligned}
$$

where

$$
M=\frac{\left(\gamma_{a}+\mu_{a}\right)\left(\alpha_{a}+\mu_{a}\right)\left(\gamma_{H}+\mu_{H}\right)\left(\alpha_{H}+\mu_{H}\right) \omega}{\gamma_{H} \gamma_{a} \rho \beta_{4}} .
$$

From (28), right eigenvectors $w=\left(w_{i}\right)^{T}$, where $i=1,2$, ..., 7 are

$$
\begin{aligned}
& w_{5}=\frac{\omega\left(\alpha_{a}+\mu_{a}\right) w_{7}}{\gamma_{a} \rho}, \\
& w_{6}=\frac{\omega w_{7}}{\rho},
\end{aligned}
$$$$
w_{3}=\left(\frac{\omega\left(\gamma_{a}+\mu_{a}\right)\left(\alpha_{a}+\mu_{a}\right)-\gamma_{a}\left(\omega \beta_{5}+\rho \beta_{6}\right)}{\gamma_{a} \rho \beta_{4}}\right) w_{7},
$$$$
w_{4}=\left(\frac{\omega\left(\gamma_{a}+\mu_{a}\right)\left(\alpha_{a}+\mu_{a}\right)-2\left(\omega \beta_{5}+\rho \beta_{6}\right) \gamma_{a}}{\gamma_{a} \mu_{a} \rho}\right) w_{7},
$$$$
w_{1}=-\left(\frac{\omega M_{1}+\left(\omega \beta_{5}+\rho \beta_{6}\right)+\gamma_{a} \beta_{4}\left(\omega \beta_{2}+\rho \beta_{3}\right)}{\beta_{4} \gamma_{a} \mu_{H} \rho}\right) w_{7},
$$$$
w_{2}=\left(\frac{\omega\left(\gamma_{a}+\mu_{a}\right)\left(\alpha_{a}+\mu_{a}\right)-\gamma_{a}\left(\omega \beta_{5}+\rho \beta_{6}\right)\left(\alpha_{H}+\mu_{H}\right)}{\gamma_{H} \gamma_{a} \beta_{4} \rho}\right) w_{7}
$$

for which $w_{7}>0$ is free right eigenvector,

$$
\text { where } M_{1}=\left(\gamma_{a}+\mu_{a}\right)\left(\alpha_{a}+\mu_{a}\right) \text {. }
$$

The left eigenvectors $v=\left(v_{i}\right)^{T}$, where $i=1,2, \ldots, 7$ are

$$
\begin{aligned}
& v_{1}=v_{4}=0, \\
& v_{2}=\frac{\gamma_{H} v_{3}}{\gamma_{H}+\mu_{H}}, \\
& v_{5}=\left(\frac{\left(\gamma_{H}+\mu_{H}\right)\left(\alpha_{H}+\mu_{H}\right)-\gamma_{H} \beta_{2}}{\beta_{4}\left(\gamma_{H}+\mu_{H}\right)}\right) v_{3}, \\
& v_{6}=\left(\frac{\left(\gamma_{a}+\mu_{a}\right)\left(\left(\gamma_{H}+\mu_{H}\right)\left(\alpha_{H}+\mu_{H}\right)-\gamma_{H} \beta_{2}\right)}{\gamma_{a} \beta_{4}\left(\gamma_{H}+\mu_{H}\right)}\right) v_{3}, \\
& v_{7}=\left(\frac{\gamma_{H} \beta_{3} \beta_{4}+\beta_{6}\left(\left(\gamma_{H}+\mu_{H}\right)\left(\alpha_{H}+\mu_{H}\right)-\gamma_{H} \beta_{2}\right)}{\omega\left(\gamma_{H}+\mu_{H}\right) \beta_{4}}\right) v_{3},
\end{aligned}
$$

for which $v_{3}>0$ is free left eigenvector.

3.5.1. Computation of $\boldsymbol{a}$. From the model system (4), the associated nonzero partial derivatives of $F$ at disease free equilibrium are given by 


$$
\begin{aligned}
& \frac{\partial^{2} f_{2}}{\partial x_{3}^{2}}=-\frac{2 \beta_{1} \mu_{H}}{\Lambda_{H}}, \\
& \frac{\partial^{2} f_{2}}{\partial x_{2} \partial x_{3}}=-\frac{\beta_{1} \mu_{H}}{\Lambda_{H}}, \\
& \frac{\partial^{2} f_{2}}{\partial x_{2} \partial x_{6}}=-\frac{\beta_{2} \mu_{H}}{\Lambda_{H}}, \\
& \frac{\partial^{2} f_{2}}{\partial x_{2} \partial x_{7}}=\frac{-\beta^{*} \mu_{H}}{\Lambda_{H}}, \\
& \frac{\partial^{2} f_{2}}{\partial x_{3} \partial x_{6}}=-\frac{\beta_{2} \mu_{H}}{\Lambda_{H}}, \\
& \frac{\partial^{2} f_{2}}{\partial x_{3} \partial x_{7}}=\frac{-\beta^{*} \mu_{H}}{\Lambda_{H}}, \\
& \frac{\partial^{2} f_{5}}{\partial x_{6}^{2}}=-\frac{2 \beta_{5} \mu_{a}}{\Lambda_{a}}, \\
& \frac{\partial^{2} f_{5}}{\partial x_{3} \partial x_{6}}=-\frac{\beta_{4} \mu_{a}}{\Lambda_{a}}, \\
& \frac{\partial^{2} f_{5}}{\partial x_{5} \partial x_{6}}=\frac{-\beta_{5} \mu_{a}}{\Lambda_{a}}, \\
& \frac{\partial^{2} f_{5}}{\partial x_{5} \partial x_{7}}=\frac{-\beta_{6} \mu_{a}}{\Lambda_{a}}, \\
& \frac{\partial^{2} f_{5}}{\partial x_{6} \partial x_{7}}=\frac{-\beta_{6} \mu_{a}}{\Lambda_{a}}, \\
& \frac{\partial^{2} f_{5}}{\partial x_{3} \partial x_{5}}=-\frac{2 \beta_{4} \mu_{a}}{\Lambda_{a}} .
\end{aligned}
$$

Since $v_{1}=v_{2}=0$, it follows that

$$
a=v_{2} \sum_{i, j=1}^{n} w_{i} w_{j} \frac{\partial^{2} f_{2}}{\partial x_{i} \partial x_{j}}+v_{5} \sum_{i, j=1}^{n} w_{i} w_{j} \frac{\partial^{2} f_{5}}{\partial x_{i} \partial x_{j}} .
$$

To compute $a$, we substitute the partial derivatives into (35) to obtain

$$
\begin{aligned}
a= & \left(\frac{2 \mu_{H} \Lambda_{H} w_{3} M_{2} \beta_{1} \gamma_{a}\left(\omega \beta_{5}+\rho \beta_{6}\right)}{\Lambda_{H} \gamma_{H} \Lambda_{a} \gamma_{a} \rho \beta_{4}}\right) w_{7} v_{2} \\
& -\left(\frac{2 \mu_{H} \Lambda_{a} w_{3} M_{1} \beta_{1} \omega M_{1}+\gamma_{a} \beta_{4}\left(\omega \beta_{2}+\rho \beta_{3}\right)}{\Lambda_{H} \gamma_{H} \Lambda_{a} \gamma_{a} \rho \beta_{4}}\right) w_{7} v_{2} \\
& -\left(\frac{2 \Lambda_{H} \gamma_{H} \omega \beta_{4} \mu_{a}\left(w_{5}+w_{6}\right) M_{1}}{\Lambda_{H} \gamma_{H} \Lambda_{a} \gamma_{a} \rho \beta_{4}}\right) w_{7} v_{5},
\end{aligned}
$$

where $M_{2}=\alpha_{H}+\mu_{H}+\gamma_{H}$.

To analyze the sign of $a$, we consider two cases.

Case I:

$a<0$ if

$$
\begin{array}{r}
\frac{\gamma_{a}\left(\omega \beta_{5}+\rho \beta_{6}\right)}{\omega\left(\gamma_{a}+\mu_{a}\right)\left(\alpha_{a}+\mu_{a}\right)} \\
\frac{\Lambda_{H} \gamma_{H} \mu_{a} \omega \beta_{4}\left(w_{5}+w_{6}\right) M_{1} v_{5}+\Lambda_{a} \mu_{H} w_{3}\left(\omega M_{1} \beta_{1}+\left(\omega \beta_{2}+\rho \beta_{3}\right) \gamma_{a} \beta_{4}\right) M_{1} v_{2}}{\Lambda_{H} \gamma_{a} \mu_{H} M_{2} \beta_{1}\left(\omega \beta_{5}+\rho \beta_{6}\right) w_{3} v_{2}}>1 .
\end{array}
$$

Case II:

$a>0$ if

$$
\frac{\gamma_{a}\left(\omega \beta_{5}+\rho \beta_{6}\right)}{\omega\left(\gamma_{a}+\mu_{a}\right)\left(\alpha_{a}+\mu_{a}\right)}>1
$$

$\frac{\Lambda_{H} \gamma_{H} \mu_{a} \omega \beta_{4}\left(w_{5}+w_{6}\right) M_{1} v_{5}+\Lambda_{a} \mu_{H} w_{3}\left(\omega M_{1} \beta_{1}+\left(\omega \beta_{2}+\rho \beta_{3}\right) \gamma_{a} \beta_{4}\right) M_{1} v_{2}}{\Lambda_{H} \gamma_{a} \mu_{H} M_{2} \beta_{1}\left(\omega \beta_{5}+\rho \beta_{6}\right) w_{3} v_{2}}<1$. 
TABLE 4: Parameter values of the model system 3.1.

\begin{tabular}{lccc}
\hline Parameter & Interpretation & Value $y r^{-1}$ & Source \\
\hline$\Lambda_{H}$ & Human recruitment rate & 36 & {$[16]$} \\
$\beta_{1}$ & Human infection rate from infected human & 0.35 & Estimated \\
$\beta_{2}$ & Human infection rate from infected animals & 0.55 & \\
$\beta_{3}$ & Human infection rate from infected dairy products & 0.999 & Estimated \\
$\beta_{4}$ & Rate of cow infected via human & 0.25 & Estimated \\
$\beta_{5}$ & Rate of cow infected via animal & 0.6 & {$[15]$} \\
$\Lambda_{a}$ & Animal recruitment rate & 200 & 0.34 \\
$\beta_{6}$ & Rate of animals infected via dairy products & 0.01 & \\
$\mu_{H}$ & Human natural death rate & 0.139 & \\
$\alpha_{H}$ & Human death rate due to disease induced & 0.18 & \\
$\gamma_{a}$ & Progression rate from $E_{a}$ to $I_{a}$ & 0.0304 & {$[15]$} \\
$\alpha_{a}$ & Animal death due to disease induced & 0.18 & {$[16]$} \\
$\gamma_{H}$ & Progression rate from $E_{H}$ to $I_{H}$ & 0.05 & \\
$\mu_{a}$ & Animal natural death rate & 0.69 & Estimated \\
$\rho$ & Dairy production rate & 0.4 & Estimated \\
$\omega$ & Rate of decaying dairy products & {$[30]$} \\
\hline
\end{tabular}

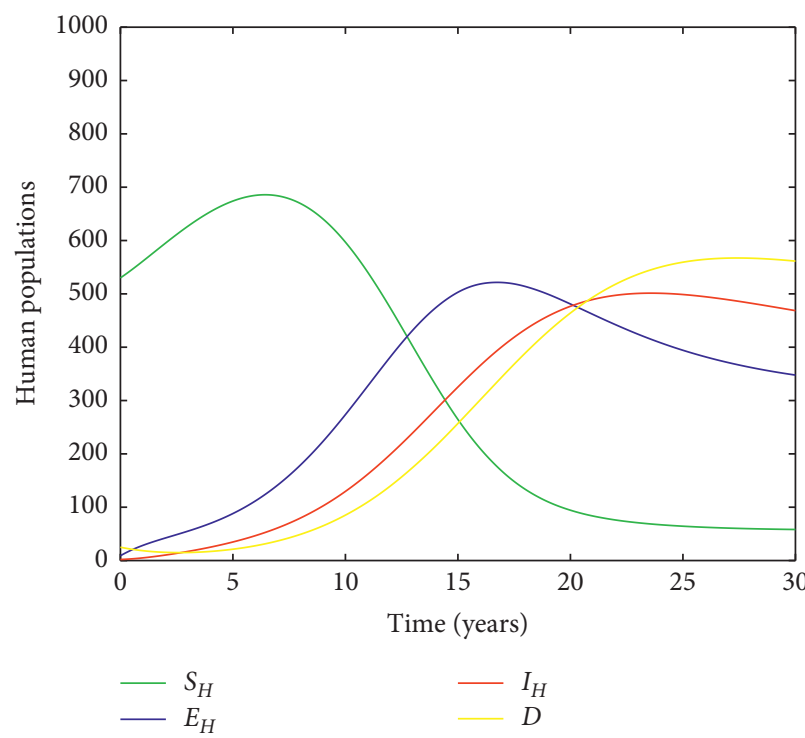

(a)

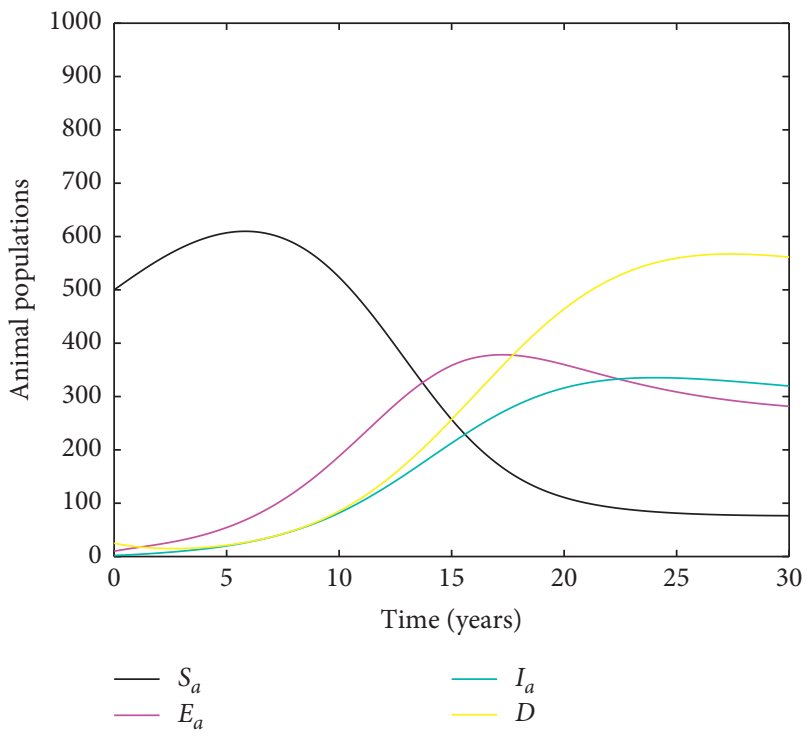

(b)

Figure 2: Dynamics of bTB in (a) human population and (b) animal population.

3.5.2. Computation of $\boldsymbol{b}$. Recall from (33), since $v_{1}=v_{4}=0$ $b$ becomes

$$
\begin{aligned}
& b=v_{2} \sum_{i=1}^{n} w_{i} \frac{\partial^{2} f_{k}}{\partial x_{i} \partial \beta^{*}}(0,0), \\
& b=v_{2} w_{7} \frac{\partial^{2} f_{2}}{\partial x_{7} \partial \beta^{*}} \\
& b=\frac{\gamma_{H} w_{7} v_{3}}{\gamma_{H}+\mu_{H}}>0 .
\end{aligned}
$$

From the computation of $a$ and $b$, we can establish the following results.

Theorem 3. If condition (38) holds, bTB undergoes backward bifurcation at $R_{0}=1$. This implies that if $a>0, b>0$ when $\beta^{*}<0$, DFE is locally asymptotically stable and there exist a positive unstable equilibrium and when $\beta^{*}>0$, and DFE is unstable and there exist a negative and locally asymptotically stable equilibrium.

\section{Numerical Simulation}

In this section, we discuss the dynamics of bTB in humans and animal population by considering parameters which drive the transmission dynamics of bTB. We use estimated parameters and some from the related literature as summarized in Table 4.

Susceptible humans and animals decrease after acquiring bTB when they come into contact with infectious humans and animals and after consuming infectious dairy products, as shown in Figure 2. However, infectious classes increase as 


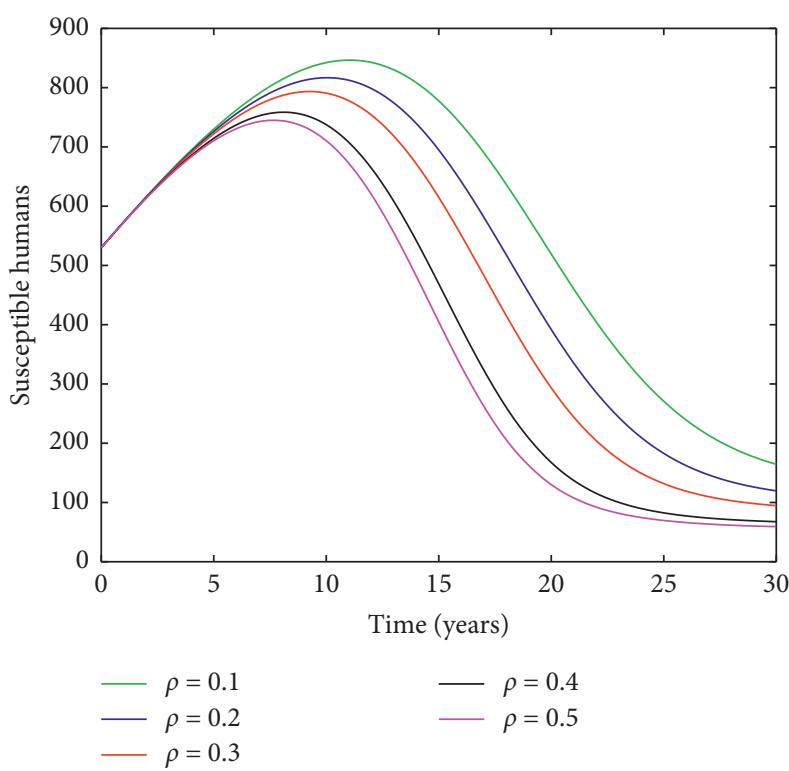

(a)

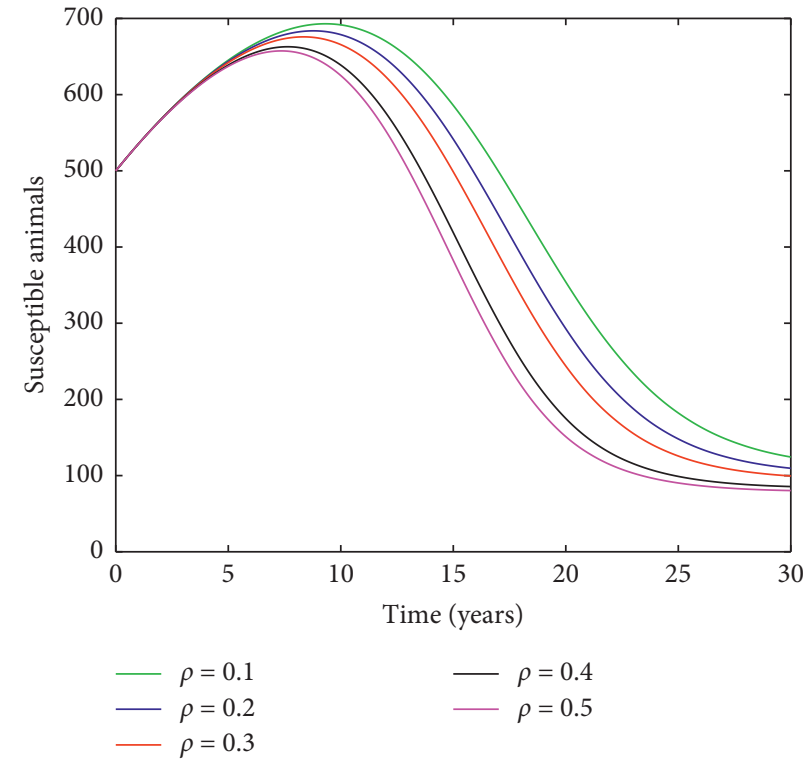

(b)

Figure 3: Variation of the dairy production rate into susceptible (a) humans and (b) animals with change in time.

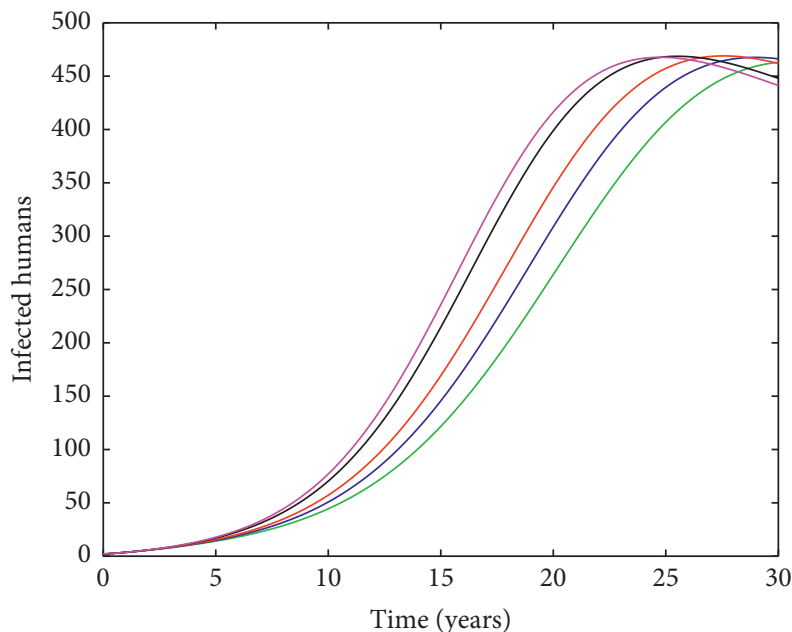

$\begin{aligned} \rho & =0.1 \\ -\rho & =0.2 \\ \rho & =0.3\end{aligned}$

(a)

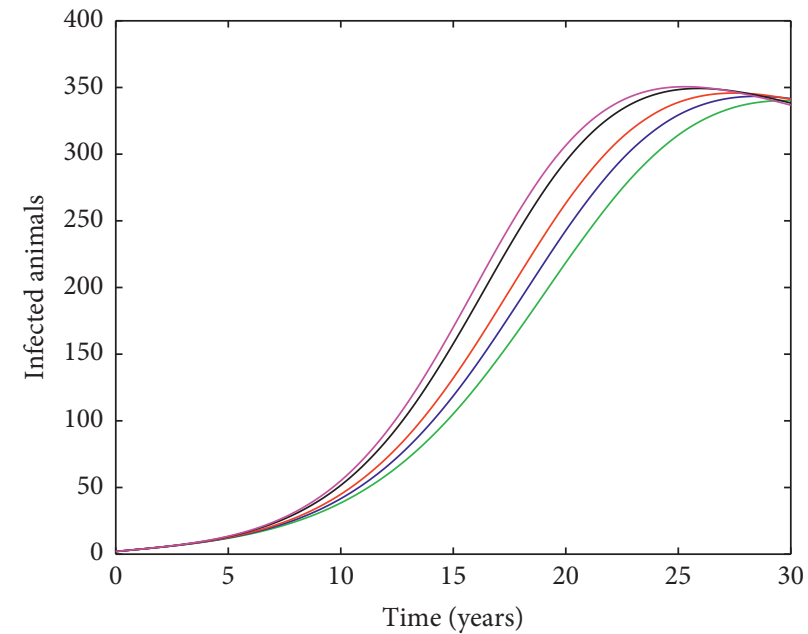

(b)

FIgURE 4: Variation of the dairy production rate into infected (a) humans and (b) animals with change in time.

individuals from susceptible class acquire bTB and move to exposed class and then to infectious class.

Figures 3(a) and 3(b) show the variations of susceptible humans and animals as rates of consuming dairy products increase. As dairy products from infectious animals increase, their consumption increases thus increasing the bTB infection rate for susceptible humans and animals.

As we increase the rate of producing infectious dairy products, susceptible classes decrease and infectious classes increase. This is due to the fact that the more dairy products are produced the more susceptible humans and animals consume the products and get infection, thus replenish infectious classes, as demonstrated in Figure 4.

Figure 5 shows the effects of varying human and animal transmission rates from infectious animals. If effective control measures are not taken to contain the transmission, infectious class increases as humans and animals come in contact with infectious animals. When the rate of contact increases, susceptible human and animal classes decrease, as shown in Figure 5. 

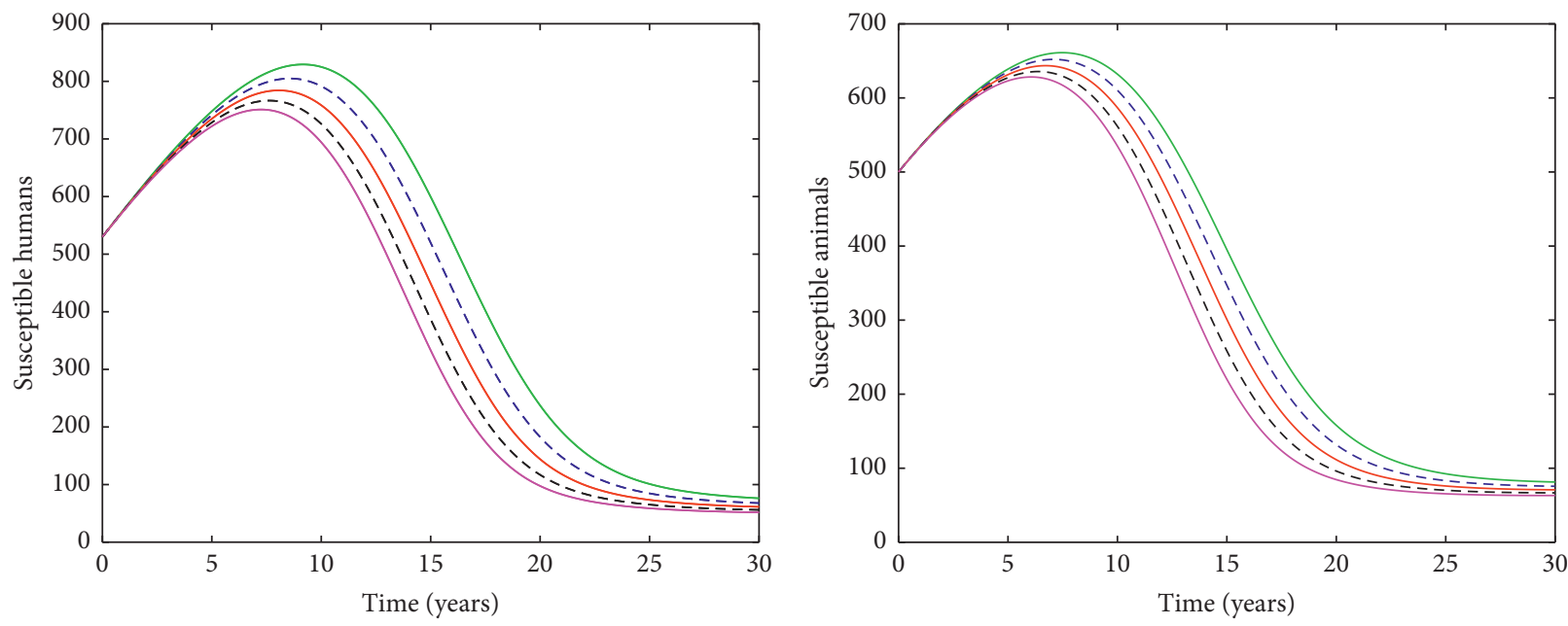

$\begin{aligned}-\beta_{2}=0.1 & --\beta_{2}=0.7 \\ --\beta_{2}=0.3 & -\beta_{3}=0.9 \\ - & \beta_{2}=0.5\end{aligned}$

(a)

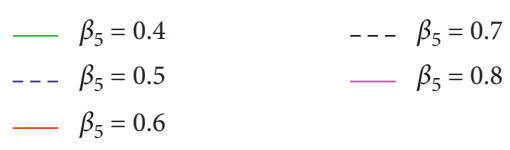

(b)

FIGURE 5: Variation of humans and animals infection rates from infectious animals to susceptible humans and animals classes. (a) Susceptible humans. (b) Susceptible animals.

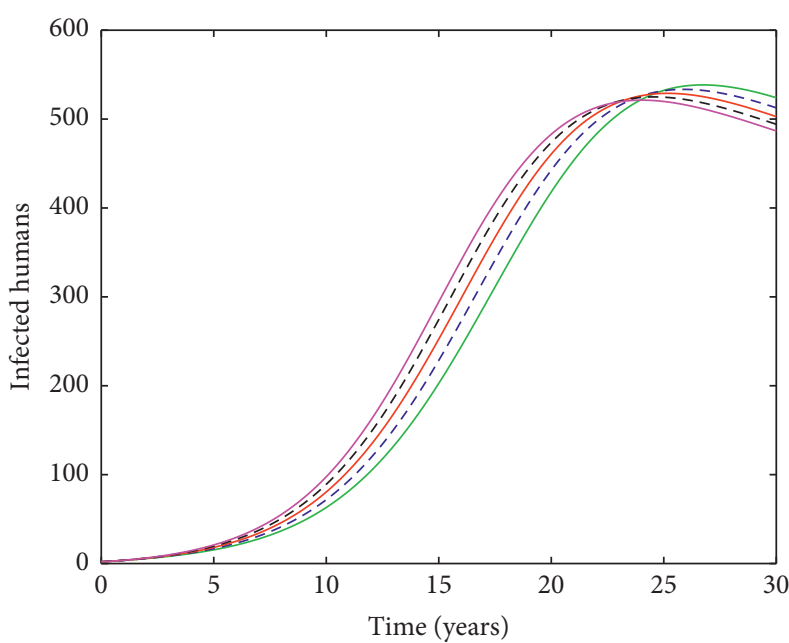

$$
\begin{array}{ll}
-\beta_{2}=0.1 & --\beta_{2}=0.7 \\
--\beta_{2}=0.3 & -\beta_{3}=0.9 \\
- & \beta_{2}=0.5
\end{array}
$$

(a)

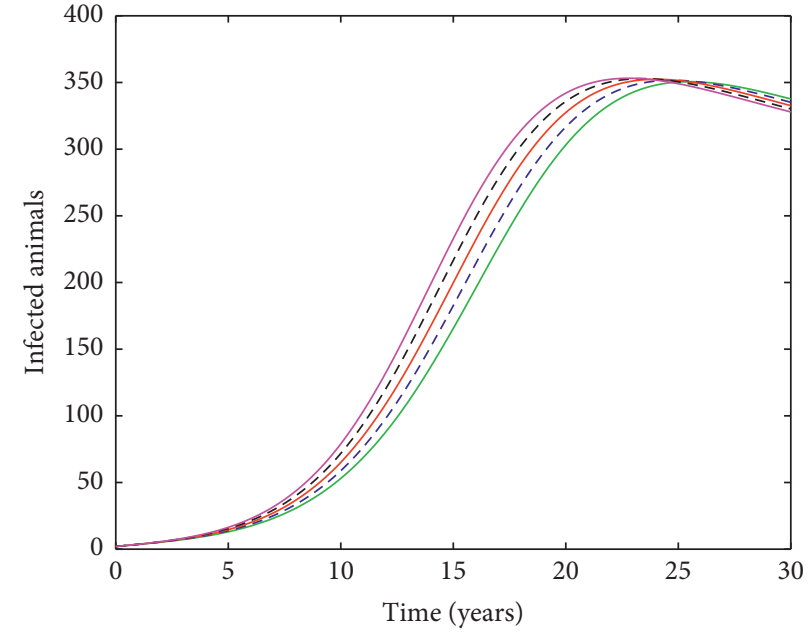

$$
\begin{array}{ll}
-\beta_{5}=0.4 & --\beta_{5}=0.7 \\
--\beta_{5}=0.5 & -\beta_{5}=0.8 \\
-\beta_{5}=0.6 &
\end{array}
$$

(b)

FIGURE 6: Variation of humans and animals infection rates from infectious animals to infectious human and animals classes, respectively. (a) Infected humans. (b) Infected animals.

Infectious human and animals classes increase over time as we vary infection rates. This is due to the fact that the more interaction between susceptible humans and animals with infectious animals increases infection rate, as shown in Figure 6.

Figure 7 shows the impacts of transmission rates from infectious dairy products $\beta_{3}$ and $\beta_{6}$ to susceptible human and animal classes. The graphs show that the increase in production of infectious dairy products increases proportionally to the transmission rates. As rates of transmission $\beta_{3}$ and $\beta_{6}$ increase, susceptible humans and animals decrease, as shown in Figure 7.

Figures 8(a) and 8(b) show how the increase in the transmission rate from dairy products to susceptible humans 


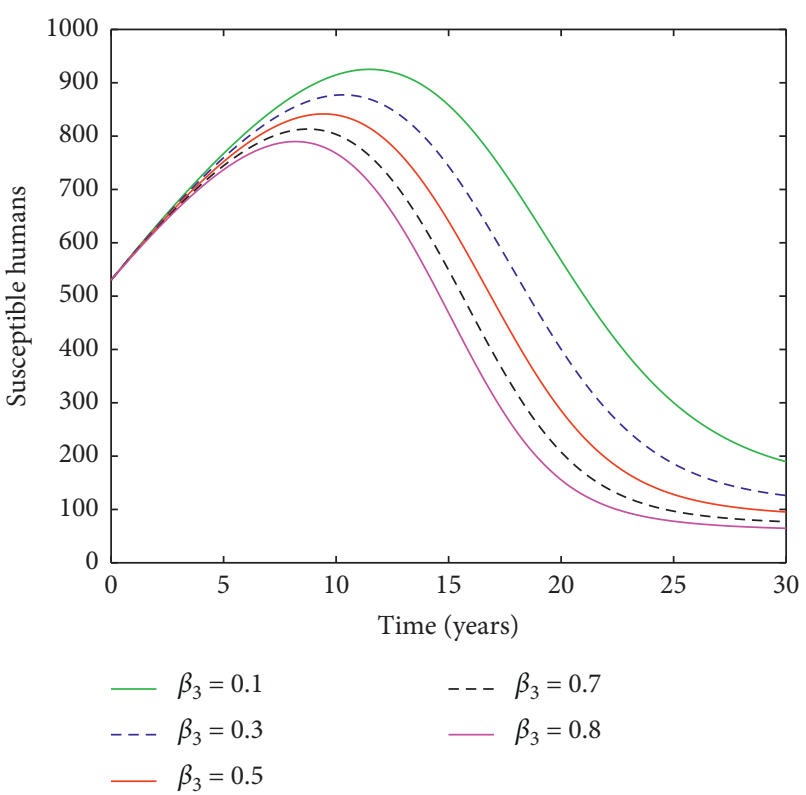

(a)

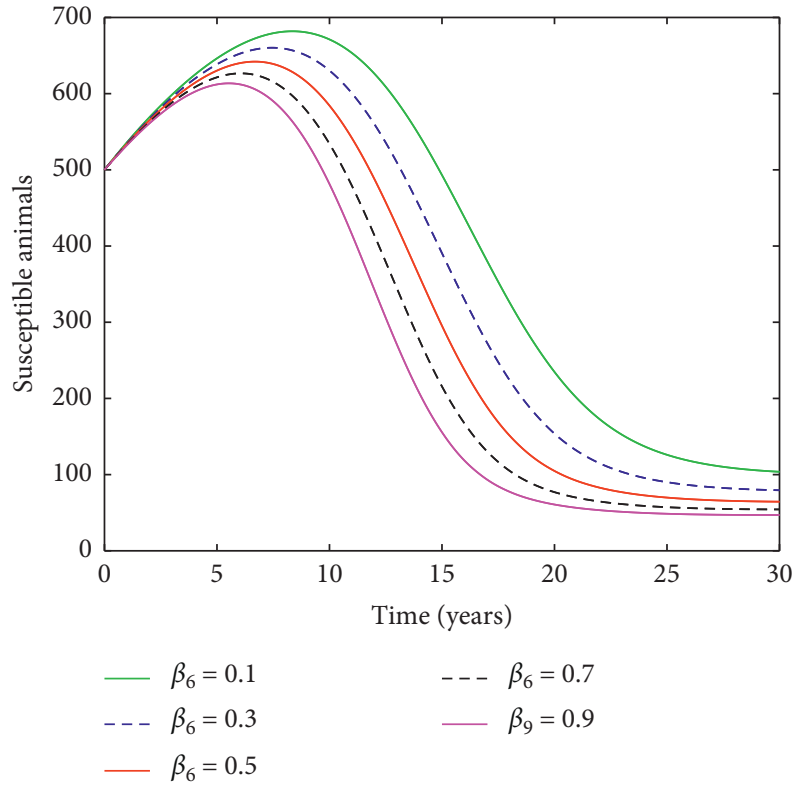

(b)

Figure 7: The impact of rates of transmission form dairy products to susceptible (a) humans and (b) animals.

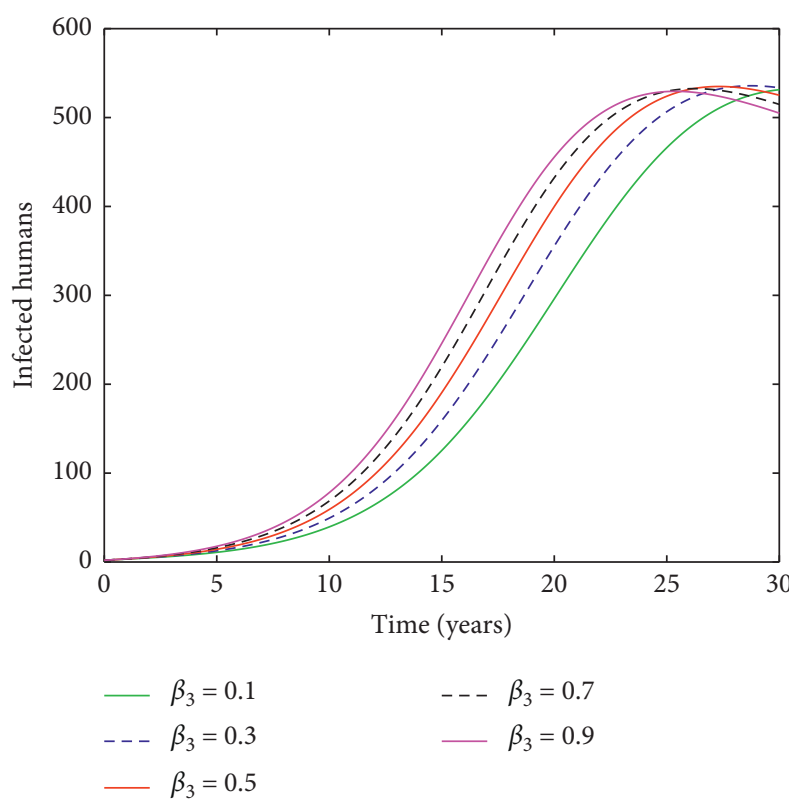

(a)

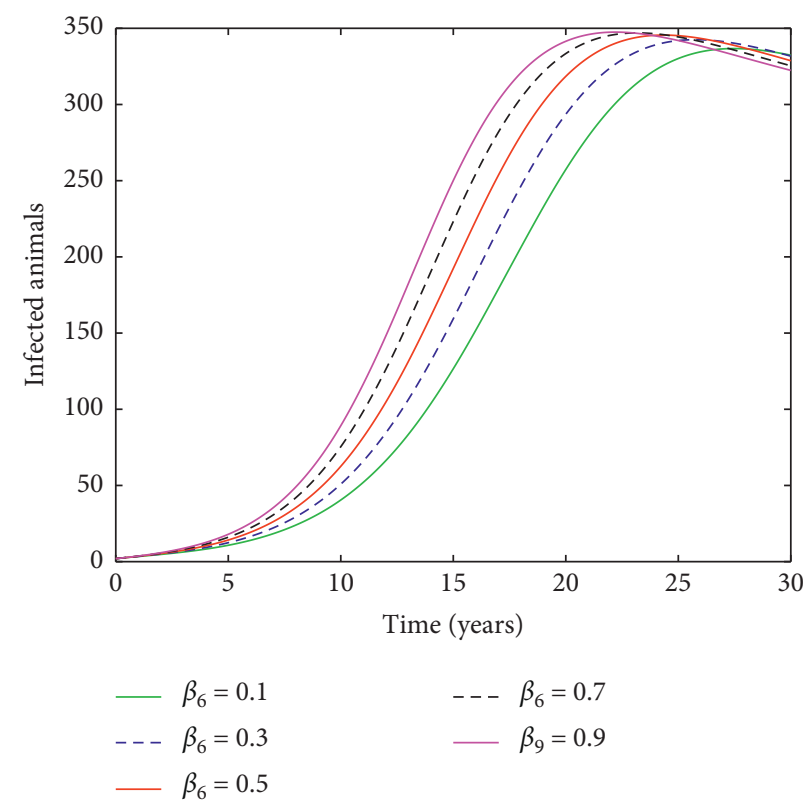

(b)

FIgURE 8: The impact of rates of transmission form dairy products to infected (a) humans and (b) animals.

$\beta_{3}$ and animals $\beta_{6}$ decrease susceptible classes, which then increase infectious classes.

\section{Conclusion and Recommendations}

A deterministic model for transmission dynamics of bTB is developed and analyzed to determine parameters that drive the disease. We computed basic reproduction number $R_{0}$ and compute the sensitivity index of each parameter with respect to $R_{0}$. Analysis shows that the animal infection rate from infectious animals $\beta_{5}$, production of infectious dairy products $\rho$, human infection rate from dairy products $\beta_{3}$, and humans infection rate from infectious animals $\beta_{2}$ drive the dynamics of bTB. Stability of equilibrium states was investigated, disease-free equilibrium DFE is locally asymptotically stable when the basic reproduction number $R_{0}$ $<1$. However, both disease-free and endemic equilibria are not globally stable due to possibility of the model to undergo 
backward bifurcation when the basic reproduction number $R_{0}=1$. To contain the disease, control strategies should target to reduce animal infection rate, production of infectious dairy products, infection rate from dairy products, and infection rate from infectious animals. We recommend quarantine of infected animals, inspection of meat, pasteurizing of milk, and education campaign to reduce contact between humans and animals.

\section{Data Availability}

The data used to support the findings of this study are mainly from the literature similar to this study, where unavailable data especially values of parameters were estimated for the purpose of verifying results of the mathematical analysis of the model.

\section{Conflicts of Interest}

The authors have no conflicts of interest to declare.

\section{Acknowledgments}

The authors wish to express sincere gratitude to the management of Sokoine University of Agriculture (Department of Veterinary Medicine) for their strong cooperation during the study. Appreciation also goes to Dr. Gabriel M. Shirima and Mr. Nkuba Nyerere for their advice and assistance during data analysis. Finally, we thank African Development Bank for funding this study.

\section{References}

[1] W. H. Organization, Global Tuberculosis Report 2018, World Health Organization, Geneva, Switerland, 2018.

[2] WHO, Global Tuberculosis Report 2017, World health organization, Geneva, Switerland, 2016.

[3] L. Durnez, A. Katakweba, H. Sadiki et al., "Mycobacteria in terrestrial small mammals on cattle farms in tanzania," Veterinary Medicine International, vol. 2011, 2011.

[4] M. De Garine-Wichatitsky, A. Caron, R. Kock et al., "A review of bovine tuberculosis at the wildlife-livestock-human interface in sub-Saharan Africa," Epidemiology and Infection, vol. 141, no. 7, pp. 1342-1356, 2013.

[5] S. W. Dejene, I. M. Heitkönig, H. H. Prins et al., "Risk factors for bovine tuberculosis (bTB) in cattle in Ethiopia," PLoS One, vol. 11, no. 7, pp. 1-16, 2016.

[6] B. Z. Katale, E. V. Mbugi, E. D. Karimuribo et al., "Prevalence and risk factors for infection of bovine tuberculosis in indigenous cattle in the Serengeti ecosystem, Tanzania," BMC Veterinary Research, vol. 9, 2013.

[7] F. Menzies and S. Neill, "Cattle-to-cattle transmission of bovine tuberculosis," The Veterinary Journal, vol. 160, no. 2, pp. 92-106, 2000.

[8] B. Raymond, K. L. Wyres, S. K. Sheppard, R. J. Ellis, and M. B. Bonsall, "Environmental factors determining the epidemiology and population genetic structure of the bacillus cereus group in the field," PLoS Pathogens, vol. 6, no. 5, Article ID e1000905, 2010.

[9] G. M. Shirima, R. R. Kazwala, and D. M. Kambarage, "Prevalence of bovine tuberculosis in cattle in different farming systems in the eastern zone of Tanzania," Preventive Veterinary Medicine, vol. 57, no. 3, pp. 167-172, 2003.

[10] A. M. Kilale, "Mycobacteria in Northern Tanzania: exposure and risk of disease among agropastoralists and programmatic challenges in investigation of re-treatment cases," 2016.

[11] F. Mathews, D. W. Macdonald, G. M. Taylor et al., "Bovine tuberculosis (Mycobacterium bovis) in British farmland wildlife: the importance to agriculture," Proceedings of the Royal Society B: Biological Sciences, vol. 273, no. 1584, pp. 357-365, 2006.

[12] OIE, "Bovine tuberculosis," OIE Terrestrial Maual, vol. 2015, 2016.

[13] M. J. H. O’Hagan, E. A. Courcier, J. A. Drewe, A. W. Gordon, J. McNair, and D. A. Abernethy, "Risk factors for visible lesions or positive laboratory tests in bovine tuberculosis reactor cattle in Northern Ireland," 2013.

[14] F. Biet, M. L. Boschiroli, M. F. o. Thorel, and L. A. Guilloteau, "Zoonotic aspects of mycobacterium bovis and mycobacterium avium-intracellulare complex (mac)," Veterinary Research, vol. 36, no. 3, pp. 411-436, 2005.

[15] F. B. Agusto, S. Lenhart, A. B. Gumel, and A. Odoi, "Mathematical analysis of a model for the transmission dynamics of bovine tuberculosis," Mathematical Methods in the Applied Sciences, vol. 34, no. 15, pp. 1873-1887, 2011.

[16] S. Liu, A. Li, X. Feng, X. Zhang, and K. Wang, "A dynamic model of human and livestock tuberculosis spread and control in Urumqi, Xinjiang, China," Computational and Mathematical Methods in Medicine, vol. 2016, 2016.

[17] P. B. Phepa, Submitted in Fulfilment of the Degree of Doctor of Philosophy at University of KwaZulu?, 2015.

[18] O. A. Bonsu, E. Laing, and B. D. Akanmori, "Prevalence of tuberculosis in cattle in the dangme-west district of Ghana, public health implications," Acta Tropica, vol. 76, no. 1, pp. 9-14, 2000.

[19] A. M. Perez, M. P. Ward, A. Charmandarián, and V. Ritacco, "Simulation model of within-herd transmission of bovine tuberculosis in argentine dairy herds," Preventive Veterinary Medicine, vol. 54, no. 4, pp. 361-372, 2002.

[20] D. F. Ramos, P. E. A. Silva, and O. A. Dellagostin, "Diagnosis of bovine tuberculosis: review of main techniques," Brazilian Journal of Biology, vol. 75, no. 4, pp. 830-837, 2015.

[21] W. Assembly, "Bovine tuberculosis techniques," 2009.

[22] O. Diekmann, J. A. P. Heesterbeek, and J. A. Metz, "On the definition and the computation of the basic reproduction ratio in models for infectious diseases in heterogeneous populations," Journal of Mathematical Biology, vol. 28, no. 4, pp. 365-382, 1990.

[23] J. I. Irunde, L. S. Luboobi, and Y. Nkansah-Gyekye, "Modeling the effect of tobacco smoking on the in-host dynamics of hiv/ aids," Journal of Mathematical and Computational Science, vol. 6, no. 3, p. 406, 2016.

[24] P. Van den Driessche and J. Watmough, "Reproduction numbers and sub-threshold endemic equilibria for compartmental models of disease transmission," Mathematical Biosciences, vol. 180, no. 1-2, pp. 29-48, 2002.

[25] W. Fellin, H. Lessmann, M. Oberguggenberger, and R. Vieider, Analyzing Uncertainty in Civil Engineering, Springer, Berlin, Germany, 2005.

[26] C. J. Silva and D. F. M. Torres, "Optimal control for a tuberculosis model with reinfection and post-exposure interventions," Mathematical Biosciences, vol. 244, no. 2, pp. 154-164, 2013.

[27] N. Chitnis, J. M. Hyman, and J. M. Cushing, "Determining important parameters in the spread of malaria through the 
sensitivity analysis of a mathematical model," Bulletin of Mathematical Biology, vol. 70, no. 5, pp. 1272-1296, 2008.

[28] C. Castillo-Chavez and B. Song, "Dynamical models of tuberculosis and their applications," Mathematical Biosciences and Engineering, vol. 1, no. 2, pp. 361-404, 2004.

[29] A. S. Hassan, S. M. Garba, A. B. Gumel, and J.-S. Lubuma, "Dynamics of mycobacterium and bovine tuberculosis in a human-buffalo population," Computational and Mathematical Methods in Medicine, vol. 2014, 2014.

[30] A. Ssematimba, J. Jores, and J. C. Mariner, "Mathematical modelling of the transmission dynamics of contagious bovine pleuropneumonia reveals minimal target profiles for improved vaccines and diagnostic assays," PLoS One, vol. 10, no. 2, pp. 1-15, 2015. 